\title{
Tics and functional tic-like movements
}

Can we tell them apart?

Christos Ganos, MD, Davide Martino, MD, PhD, Alberto J. Espay, MD, Anthony E. Lang, MD, FRCPC, Kailash P. Bhatia, FRCP, and Mark J. Edwards, MD, PhD

Neurology ${ }^{\circledR}$ 2019;93:1-9. doi:10.1212/WNL.0000000000008372

\section{Abstract}

Within the broad spectrum of movement disorders, tics and functional tic-like movements belong to a particular clinical category. Both types of movements are within the range of normal movement kinematics and muscle synergies, but appear repetitive and without appropriate context embedment. Historically, there have been many attempts to separate the 2 types of movements, but because of their phenomenological overlap, clinical distinction may be prone to error, and misdiagnoses may often occur. Most importantly, the 2 types of movement may coexist. Here, we review the available literature on the 2 types of motor phenomena and demonstrate some of the difficulties in distinguishing tics from functional tic-like movements on clinical grounds. We also highlight similarities and differences in pathophysiologic characteristics, documenting the significance of action monitoring, attentional allocation, and behavioral reinforcement in both types of movements, as well as in their risk factors. We discuss the overlap of current behavioral treatments for tics and functional tic-like movements and emphasize implications of diagnostic mislabeling. Such implications include the need to tailor behavioral treatment approaches to individual phenomenological profiles and guiding decision making for severe patients requiring invasive interventions, such as deep brain stimulation. A deeper insight from clinicians with respect to persisting challenges in classifying and differentiating these motor phenomena could accelerate the development of reliable clinical and physiologic markers (i.e., next generation phenotyping) and a neurobiology-driven therapeutic approach for these motor phenomena.
Correspondence

Dr. Ganos

christos.ganos@charite.de

From the Department of Neurology (C.G.), Charité University Medicine Berlin, Germany; Department of Clinical Neurosciences (D.M.), Cumming School of Medicine, University of Calgary and Hotchkiss Brain Institute, AB, Canada; Department of Neurology (A.J.E.), James J. and Joan A. Gardner Family Center for Parkinson's Disease and Movement Disorders, University of Cincinnati, OH; Division of Neurology (A.E.L.), Edmond J. Safra Program in Parkinson's Disease Morton and Gloria Shulman Movement Disorders Clinic, Toronto Western Hospital-UHN, University of Toronto, ON, Canada; Department of Clinical and Movement Neurosciences (K.P.B.), Queen Square Institute of Neurology, University College London; and Neurosciences Research Centre (M.E.), Molecular and Clinical Sciences Institute, St George's University of London.

Go to Neurology.org/N for full disclosures. Funding information and disclosures deemed relevant by the authors, if any, are provided at the end of the article. 


\section{Glossary}

CBI = cognitive behavioral intervention; DBS = deep brain stimulation; $\mathbf{T S}=$ Tourette syndrome.

Tics represent a rather particular category of movement disorders. Rather than being an unnatural, involuntary, and nonphysiologic movement pattern (as seen in dystonia or myoclonus for example), tics are within the range of volitional movement kinematics and muscle synergies. ${ }^{1}$ The abnormality is instead at the level of control, that is, whether and when a movement will occur.

Historically, tics have been separated from another disorder also characterized as a problem of willed control over movement: functional/psychogenic movement disorders. ${ }^{2}$ However, here, we suggest that because they share phenomenological traits, it is highly likely that they may overlap and people classified as having tics may in fact have functional tic-like movements, and vice versa. Perhaps underappreciated, both movement disorders could coexist, and there are no reliable clinical or neurophysiologic biomarkers that clearly distinguish one from the other. The appreciation of the possible coexistence of tics and functional tic-like movements in the same patient should influence management approach, including selection for invasive/ experimental treatments, such as deep brain stimulation (DBS).

\section{Two syndromes or one?}

A single tic is indistinguishable from a rapid voluntary movement. ${ }^{3}$ Any biomechanically possible movement with muscle activation parameters within those accessible for normal voluntary movement may occur as a tic behavior. These same parameters can define functional tic-like movements. Therefore, on the exclusive basis of a rapid focal movement (e.g., a head jerk or a blink), in the absence of historical context, it may not be possible to clinically classify it as a voluntary action, a tic, or a functional tic-like movement. However, with longer duration of observation, the ability to distinguish these phenomena is improved. Another criterion to distinguish between the different types of motor behaviors could be the frequency of their occurrence. However, this criterion is most likely not sufficient to differentiate between tics and functional tic-like movements, as their frequency is dictated by clinical severity in both disorders. The addition of historical information including symptom reports beyond those reflected in observable motor phenomena adds another (and arguably most important) level of diagnostic information. This is perhaps why clinical research to date aimed at distinguishing between tics and functional tic-like movements has emphasized syndromic features.

\section{Current clinical framework separating tics from functional tic-like movements}

The earliest study addressing the clinical features between patients with functional tic-like movements $(\mathrm{n}=9$; "psychogenic movement disorders resembling tics") and Tourette syndrome (TS), the prototypical primary tic disorder, showed that unlike the latter, the former exhibit lack of premonitory urges and inability to suppress unwanted motor behaviors. ${ }^{4}$ Other features of functional tic-like disorders included female preponderance, the presence of additional functional movement disorders, lack of response to typical anti-tic medications, and the absence of a family history of tic disorders. A subsequent study complemented this list of clinical clues with data collected from a group of 12 patients diagnosed with functional tic-like movements. ${ }^{5}$ In these patients, the distribution of tic-like movements lacked the characteristic rostrocaudal gradient typical for tics in patients with TS. Indeed, in patients diagnosed with functional tic-like movements, the cranial region was not as commonly affected as the trunk or the extremities. Also, the functional tic-like movements commonly interfered with the execution of voluntary actions ("blocking tics") and pali-, echo-, or coprophenomena, which are characteristic although not necessarily frequent in patients with primary tic disorders and TS, were not observed. Furthermore, functional tic-like movements lacked the characteristic waxing and waning of symptoms that is nearly universally present in patients with primary tic disorders and TS, particularly at younger ages. Taken together, these 2 studies provided the basic framework to distinguish patients with functional tic-like disorders from patients with primary tic disorders on the basis of their clinical characteristics.

\section{Overlap between tics and tic-like movements}

A challenging problem is that some people exhibit features observed in both primary tics and functional tic-like disorders, similar to patients with epilepsy, who may present with both epileptic and nonepileptic seizures. ${ }^{6}$ Indeed, overlap between neurologic illness and functional neurologic symptoms is a common phenomenon across the spectrum of neurologic disorders. ${ }^{7}$ The presence of echophenomena was previously documented in 1 patient with functional tic-like movements. ${ }^{8}$ Moreover, repetitive vocalizations, including repetitive swearing, were noted in a group of 13 patients, which supported an initial diagnosis of a primary tic disorder; however, on reevaluation by TS experts, these patients were subsequently reclassified as functional. ${ }^{9}$ Of note, behaviors resembling coprolalia of functional nature were already documented by Charcot. ${ }^{2}$ We have reported an insidious childhood onset, tic-like behaviors involving the face, and ability to suppress tic-like movements in some of these patients. ${ }^{9}$ Some patients even endorsed sensory phenomena preceding tic-like movements. It is notable that sensory phenomena (e.g., pressure sensation or feeling overwhelmed with energy) are commonly reported in people with dissociative seizures and people with anxiety and panic 
disorder. ${ }^{10,11}$ Arguably, each of these clinical characteristics would support the diagnosis of a primary tic disorder. However, in all these cases, the sum of clinical features was atypical when compared with the syndromic characteristics of patients with primary tic disorders. For example, in some patients, the first symptom preceding the manifestation of tic-like behaviors was a different movement disorder, such as tremor, and in patients where voluntary suppression of tics was possible, this was associated with unusual phenomena, such as malaise, pain, or nausea. Also, the qualitative descriptions of sensory premonitions were unusual compared with reports of patients with TS (e.g., sudden energy pulse) and often did not localize to the ticcing body area. Moreover, swearing that was classified as functional coprolalia in these patients was different from the coprolalic behaviors encountered in TS. For example, although coprolalic tics are usually brief and uttered as either complete or incomplete words between sentences, and often with alterations in pitch, tone, and pronunciation, repetitive swearing in patients with functional tic-like behaviors did not have these characteristics. ${ }^{9}$ The repertoire of swearing included an unusually long list of different words or even complex sentences, which on occasion were context dependent. It should be noted that the possibility that some of these patients might have also had tics at some point in their lives was not excluded.

From the above, it becomes clear that an approach based on syndromic characteristics can be fraught with many challenges in distinguishing tics from functional tic-like movements. Moreover, patients with tics may also exhibit functional ticlike movements, and the overlay may be more common than currently recognized. Overlapping clinical features may point toward an overlap in the mechanisms that produce tics and functional tic-like movements.

\section{Convergent pathophysiology within neural pathways of volition}

In primary tic disorders, including TS, 2 pathophysiologic views dominate the literature. In 1 influential account, enhanced reinforcement learning underlies the manifestation of tic behaviors. ${ }^{12}$ Tics are the result of pathologically reinforced motor programs mediated through abnormally enhanced dopaminergic neurotransmission. ${ }^{12,13}$ In this account, tic behaviors are related to habitual behaviors. ${ }^{13} \mathrm{An}$ alternative theory suggests that tics represent unleashed movement fragments resulting from gamma-aminobutyric acid (GABA)-ergic disinhibition within the cortico-basal ganglia-thalamo-cortical loops. ${ }^{14}$ Here, the tic generator is viewed as a disinhibited and hyperexcitable loop on which some level of cortical control can modulate the onset and type of tic behavior. ${ }^{14}$ Animal models and neuropathologic studies support this hypothesis. ${ }^{14}$ Repetitive cranial and limb movements resembling human tics were generated by microinfusing $\mathrm{GABA}_{\mathrm{A}}$ receptor antagonists in the sensorimotor portion of the striatum of rodents ${ }^{15}$ and small primates $^{16}$ (striatal disinhibition model). Likewise, the unilateral disinhibition of the nucleus accumbens generated vocalizations with face validity for vocal tics. ${ }^{17}$ Of interest, the timing of tic-like movements in these disinhibition models appears to be modulated by motor cortical excitatory inputs to the striatum. ${ }^{18}$ Moreover, the limited neuropathologic evidence supports disinhibition by showing decreased numbers of inhibitory interneurons in striatal regions. ${ }^{19}$ Overall, these 2 apparently discrepant theories may in fact be complementary. Both propose that tics are generated within the neural pathways that also generate voluntary actions due to abnormally increased gain of specific neuromotor signals.

The same neural pathways are also proposed as being involved in the generation of the abnormal motor output in functional neurologic disorders. ${ }^{20}$ Abnormally strong "predictions" ${ }^{21}$ or "representations" ${ }^{20}$ of abnormal motor output enacted by an excessive allocation of attention toward the body are key pathophysiologic features. The output are movements that appear voluntary (because they require attention to manifest and are improved by distraction), but lack a sense of agency or control over them. ${ }^{20,21}$ Enhanced limbiccortical drive may specifically be involved in the selection and reinforcement of abnormal motor programs. ${ }^{20}$ Indeed, conditioning and reinforcement of abnormal patterns of movement and behavior have also been proposed in functional neurologic disorders, particularly in people with dissociative seizures. ${ }^{22}$ Such "habitual" responses can be considered similar to abnormally strong representations or predictions.

\section{Attention}

Although the aforementioned pathophysiologic models of tics and functional tic-like movements differ, current evidence suggests overlapping features. Indeed, attention is a significant factor in the expression of not only functional tic-like movements but also tics: the frequency and severity of tics increases if they become the center of attentional focus and characteristically decreases during distraction or engagement in other cognitive or physical activities. ${ }^{23,24}$ The role of attention as a prerequisite in the generation of abnormal motor output may be stronger in functional tic-like movements than in tics. Most, but not all, functional motor patterns cannot be generated without a degree of attentional allocation, ${ }^{24}$ but tics probably more commonly occur without patients attending to them, even in the complete absence of explicit awareness over their occurrence (e.g., oculomotor tics). Based on our experience, this is observed in a proportion of pediatric patients with less awareness of their tic behaviors, or at least fluctuating awareness of tics, but also in a subgroup of adult patients with long-standing history of tics, ${ }^{25}$ in whom the chronic presence of tics might have led to attenuation of their conscious perception, as they become engrained in patterns of "normal motor behaviors." ${ }^{26}$ Also, people with certain neuropsychiatric disorders where tics are prevalent, as, for example, in autism spectrum disorder, are often unaware of having tics, although they are capable of recognizing them in others. ${ }^{27}$ This is strongly contrasted with the perception of movements in functional tic-like disorders, where patients are typically 
very aware of their abnormal motor behaviors. Neurophysiologic evidence from movement-related cortical potentials also supports this view. Although movement-related cortical potentials are common to both tics and functional tic-like movements, they precede tics less commonly, ${ }^{28}$ suggested to reflect, at least partially, differences in the explicit attention allocated to the generation of these motor events. ${ }^{29}$ Behaviorally, the more attention-demanding functional tic-like movements typically interfere with and disrupt the ongoing execution of equally attention-demanding voluntary motor behaviors more often and to a greater extent than tics. 5

\section{Perception and intention}

A second important overlapping feature relates to the subjective experience of tics and functional tic-like movements. The hallmark feature of patients with functional movement disorders is the experience of abnormal movements as involuntary. ${ }^{30}$ However, labeling of motor output within the voluntary-involuntary continuum is also explicitly difficult in patients with tics, despite the fact that they can control their movements temporarily on demand. Of interest, patients with either type of movement disorder show comparable abnormalities in both their perception and experience of voluntary actions. $^{31,32}$ Delayed awareness of the voluntary intention to act has been demonstrated both for adult patients with tic disorders and functional movement disorders compared with healthy controls. This finding hints toward similarities of the cognitive mechanisms associated with monitoring of motor intentions and actions. ${ }^{31,32}$ It has been proposed that delayed awareness of volition in patients with functional movement disorders, as, for example, functional tremor, may reflect a general neurocognitive vulnerability in perceiving voluntary motor events as involuntary. ${ }^{31}$ An alternative explanation put forward is that delayed awareness of volition in adults could in fact reflect a perceptual adaptation to detect voluntary motor signals against a background of increased neuromotor noise. ${ }^{32,33}$ According to this account, in both disorders, perceptual learning of neuromotor signals related to voluntary actions could be impaired. Patients with increased levels of neuromotor noise would also experience greater impairments. Indeed, in adolescents with tic disorders, stronger experience of premonitory urges was associated with greater delay in the detection of volition. ${ }^{33}$

Importantly, difficulties in the detection of voluntary actions may also be intrinsically linked with deficit in the overall experience of volition. Indeed, both in tic disorders and functional movement disorders, impairments in the sense of agency for voluntary actions have been documented. ${ }^{34,35}$ However, different from patients with functional movement disorders, who showed reduced sense of agency over voluntary actions, ${ }^{34}$ adults with TS were found to have increased sense of agency, ${ }^{35}$ judging to be more in control of their voluntary actions than they actually were. Although the tasks to explore sense of agency that were applied were different for the 2 clinical populations, the directionality of findings could hint toward cardinal pathophysiologic similarities and differences. On the one hand, they indicate that in both disorders, there is an intrinsic disturbance of action monitoring. But, whereas in functional movement disorders, strong priors are suggested to alter the sensory consequences of actions, ${ }^{21}$ so that they are perceived as involuntary, in tic disorders, there appears to be enhanced intention attribution, so that events that are externally guided are judged as voluntary, predominantly based on the matching of intention and outcome. $^{35}$ Of note, a model where abnormally precise priors underlie the pathophysiologic basis of premonitory urges and tics has been recently proposed. ${ }^{36}$ Clinical evidence provides support for this model. Indeed, despite the variability and the lack of precision in describing urges, for every particular urge sensation, there appears to be only a very specific type of tic movement (or sound) that can satisfy it. In other words, tics may represent motor events that constitute direct responses aiming to match a specific preceding sensory experience.

Although the aforementioned differences could be attributed to several yet underexplored or even unknown factors, the age at symptom onset-typically childhood in tic disorders vs adolescence or adulthood in functional movement disorders - could be an important determinant. Indeed, the consequences of impaired action monitoring throughout the development and refinement of the voluntary motor system in children could be different from a similar impairment in adulthood. However, no longitudinal studies have explored the development of action monitoring from childhood to adulthood in patients and healthy subjects. Hence, we cannot distinguish whether the changes in action monitoring in adults with TS or functional movement disorders are the result of core pathologic mechanisms or of compensatory processes.

\section{Behavioral reinforcement}

In patients with tic disorders, strong stimulus-response associations point toward an abnormally enhanced dopaminergic reinforcement system, which in turn facilitates the formation and maintenance of novel tic behaviors. ${ }^{37}$ The same underlying mechanism might also characterize patients with functional tic-like movements, particularly cases in which the repertoire of the movement is restricted to specific effectors only (i.e., few and consistent tic-like movements over time).

\section{Shared risk factors}

The probability of developing any disorder increases with the accumulation of determinants, that is, attributes, events, or environmental factors known as risk factors. Different neuropsychiatric disorders may share common risk factors, including family history, prenatal events (e.g., maternal smoking and stress), early stressors and life events, personality profiles, and spectrum of comorbidities. In turn, overlapping risk factors but also factors that modulate disease course across disorders may indicate an overlap in pathophysiologic mechanisms. 


\section{Anxiety}

Both tic disorders and functional movement disorders are associated with a high prevalence of concurrent anxiety disorders. ${ }^{38,39}$ Clearly, anxiety disorders are also prevalent in many neuropsychiatric conditions and other movement disorders, such as Parkinson disease ${ }^{40}$ and dystonia. ${ }^{41}$ However, in tic disorders, anxiety symptoms-including social anxiety and perceived social threat-and psychosocial stressors are specifically associated with tic impairment ${ }^{42}$ and, therefore, are likely to play an important role in the exaggeration and propagation of tics. Unsurprisingly, the accumulation of major life events correlates both with tic severity and anxiety levels. ${ }^{43}$ Similarly, the somatic experience of anxiety and panic in patients with functional movement disorders following traumatic events, such as a physical injury, could precipitate functional neurologic symptoms, including movement disorders such as functional tic-like movements. ${ }^{44}$

\section{Alexithymia and obsessive-compulsive personality disorder}

The inability to identify and label emotional states in oneself and others, alongside increased obsessionality, which predisposes to patterns of mental controlling and checking at the expense of cognitive flexibility, may further reinforce abnormal beliefs and expectations and predispose to the manifestation and persistence of functional movement disorders. ${ }^{21,38}$ Although alexithymia was not found to be more common in tic disorders than healthy controls, ${ }^{45}$ it is important to note that autism spectrum disorder may coexist with a tic disor$\mathrm{der}^{27}$ and people with autism spectrum disorder are very often alexithymic. ${ }^{46}$ Notably, in both tic disorders and functional neurologic disorders, a reduced capacity to attend to bodily signals (e.g., heartbeats) has been described. ${ }^{47,48}$ This may, in turn, further contribute to perceptual sensory uncertainty, which, together with the tendency of patients from both groups to jump to conclusions, that is, making probabilistic inferences more impulsively than controls, ${ }^{49,50}$ may predispose to the development or perpetuation of abnormal motor output. In addition, the association of tics with obsessive-compulsive disorder is well established, and it may often be impossible to distinguish whether certain repetitive behaviors are tics or the results of obsessions and compulsions. ${ }^{51}$ Although there are differences between obsessivecompulsive personality disorder and obsessive-compulsive disorder, we do note the co-occurrence of mental inflexibility and obsessionality in both groups of patients.

\section{Positive family history}

Indeed, the manifestation of TS is 10-100 times more common in first-degree relatives compared with the general population. However, despite recent advances in the genetic basis of primary tic disorders, the molecular basis of tic generation remains elusive. Conversely, in patients with functional tic-like movements, family history is typically negative for tic disorders. One caveat of course is that functional movement disorders may be familial, ${ }^{52}$ so the presence or absence of family history comparing tics with functional tic- like movements is not particularly helpful. Overall, the relative contribution of genetic and shared environmental factors to the inheritability of both disorders remains uncertain.

\section{Convergence of treatment strategies}

The pharmacologic response of tics to antipsychotics has released it from interpretative psychoanalytic views. On this account, antidopaminergic treatments, the most efficacious pharmacologic agents to date, impede the process of stimulusresponse associations and thereby decrease tic severity and the development of new tics. The typical effect sizes on tic severity of antipsychotic drugs, compared with placebo, correspond to standardized mean differences approximately ranging between 0.5 and 0.75 , with inadequate evidence of superiority of one drug of this class relative to another. ${ }^{53}$ However, their efficacy is counterbalanced by a serious adverse effect profile that requires close monitoring, in many cases even leading to treatment discontinuation. Moreover, not all patients respond to these treatments or respond equally to the same dose, which could be also due to heterogeneity of effect of dopaminergic modulation on the basic pathophysiologic mechanisms of tics. Moreover, there is no evidence to date that guides appropriate patient selection for pharmacologic treatments, and polypharmacy may sometimes be the preferred solution.

Patients with functional tic-like movements characteristically lack a sustained response to classic anti-tic medication. ${ }^{4,5,9}$ There is an emerging literature on the use of behavioral methods (predominantly delivered by specialist physiotherapists and cognitive behavioral psychologists) where patients with functional movement disorders are taught competing/distracting maneuvers to prevent and stop the abnormal movements. ${ }^{54}$ Attentional diversion onto the goal of movement and away from the body itself is a key feature of such treatments. ${ }^{54}$

A very similar behavioral approach to treatment is also used in tic disorders, either in place of pharmacologic treatments (e.g., first-line treatment or on pharmacologic treatment failure) or as add-on components. Habit reversal, either as a stand-alone treatment or as part of a multicomponent cognitive behavioral intervention (CBIT), facilitates awareness of bodily signals related to tics and instructs patients to perform competing voluntary actions to block the impeding tic behavior. ${ }^{55}$ Additional behavioral treatment components of CBIT include relaxation training and functional intervention, which aim at enabling patients with tics to identify and manage precipitants and triggers that may worsen tic behaviors. ${ }^{55}$ Unsurprisingly, these components are also key therapeutic elements in the behavioral management of patients with functional movement disorders. ${ }^{54}$ Of note, approximately $50 \%$ of patients with tic disorders will respond to behavioral treatments as CBIT with comparable effect sizes to those of medication. However, the presence of anxiety disorders, and also premonitory urges, has been associated with lower tic reduction following 
nonpharmacologic interventions, including $\mathrm{CBIT}^{56} \mathrm{Al}$ though the role of premonitory urges in the occurrence and perpetuation of tic behaviors remains unclear, pharmacologic treatment of commonly occurring comorbidities, such as anxiety disorder or depression, may further augment the therapeutic success of behavioral interventions over abnormal motor behaviors, both in tic disorders and functional movement disorders.

\section{(Mis)Diagnostic implications}

Accurate diagnostic labeling in hyperkinetic tic-like disorders can be challenging, and etiologic misattribution or misdiagnoses often occur. Tics, for example, are still often viewed as the result of a "psychological disorder," and patients may be advised to undergo psychological therapies based on psychodynamic models of behavior for their "maladaptive motor output." Conversely, abnormal movements in patients with functional tic-like disorders may also be mislabeled as tics and these patients misdiagnosed with a primary tic disorder.

Although the occurrence of functional tic-like movements is relatively rare among the entire spectrum of functional movement disorders, such cases are regularly encountered in specialist tic disorders clinics. Most importantly, patients may also present with both types of movements, either for the same movement pattern-a phenomenon typically known as functional overlay - or for different motor behaviors. Such cases have been previously documented in the literature, ${ }^{57}$ and indeed, a functional etiology of acute episodic worsening of tic-like behaviors in patients with primary tics has been noted. ${ }^{58}$ Particularly noteworthy are cases with documented tic remission over adolescence and a sudden re-emergence or acute worsening of abnormal motor behaviors later in life. Crucially, the clinical characteristics of these behaviors are different at the time of re-emergence, including the associated subjective experience, their complexity, and their response to classic anti-tic pharmacology. Functional tic-like movements (or sounds), when interpreted as tics in patients with tic disorders, are usually refractory to treatments that may be effective in tics, thus leading to a clinical label of "treatment refractory tic disorder" in some patients. Such cases are of particular importance as failure of multiple treatments and dramatic clinical presentation may lead to the indication of invasive procedures such as deep brain stimulation. Reports of patients who experienced miraculous and persistent recovery of their abnormal motor behaviors following deep brain stimulation could also indicate that they had functional tic-like movements. ${ }^{59}$ Failure to recognize functional tic-like movements either as the sole clinical presentation or as functional overlay in the presence of a primary tic disorder may also have important research implications. Results from genetic studies, pathophysiologic studies, and therapeutic clinical trials may be "contaminated" by combining participants with different phenomena and/or rating tics and functional tic-like movements as if they were the same phenomenon.

\section{Conclusions: The end of the beginning?}

Here, we attempted to demonstrate some of the difficulties in distinguishing tics from functional tic-like movements. We highlighted significant overlap between clinical, pathophysiologic, and treatment characteristics of both types of movements and of shared risk factors (figure). We do not suggest that tics and functional tic-like movements are the same phenomenon. However, we do emphasize that for some cases, a clear distinction of abnormal motor behaviors within the tic/ functional tic-like movement spectrum is currently impossible. Most importantly, we emphasize that both types of movements may co-occur. Left unaddressed in this critical review, given its greater philosophical complexity, is the very framework of disease nosology: diseases "exist" or not based on features clinicians can make sense of, under the expectation that biology and pathophysiology should correspond with clinical constructs. Biology-driven phenotyping remains aspirational for even common diseases. ${ }^{60}$

At the present time, our knowledge of both tics and functional tic-like movements is insufficient to provide a solution to our diagnostic difficulties. However, we believe that it is important for the movement disorder community to accept that the issue is a real one and to recognize the potential implications. In this sense, our purpose is to shift this issue from an "unknown unknown" to a "known unknown." This allows us at the very least to be aware of the potential problems it might cause in our clinical and research practice and to do our best to ameliorate the gap, while gathering data that will help us to eventually bridge it. Clinicians should be particularly vigilant in considering the possibility of functional tic-like movements in patients with tics who are refractory to multiple interventions or who present with dramatic and overwhelming exacerbations of symptoms. The possibility of functional ticlike movements (as a "pure" phenomenon or overlay) should be actively considered when a patient is referred for invasive/ experimental treatments such as DBS. We should be cautious in weighing up the meaning of sudden and dramatic resolution of tics following DBS and be open to the possibility that the patient could have had functional tic-like movements as the predominant problem. We should use existing evidence on the clinical differentiators between tics and functional ticlike movements to guide the design of our genetic and clinical databases and clinical trials for people with tics so that we do as much as we can to avoid including people with functional tic-like movements as the sole or predominant cause of their symptoms. This process offers us the best chance to uncover the mysteries that still shroud the pathophysiology of tics and functional tic-like movements and to arrive at successful evidence-based treatments for both disorders.

\section{Author contributions}

C. Ganos: drafting/revising the manuscript; data acquisition; study concept or design; analysis or interpretation of data; accepts responsibility for conduct of research and will give 


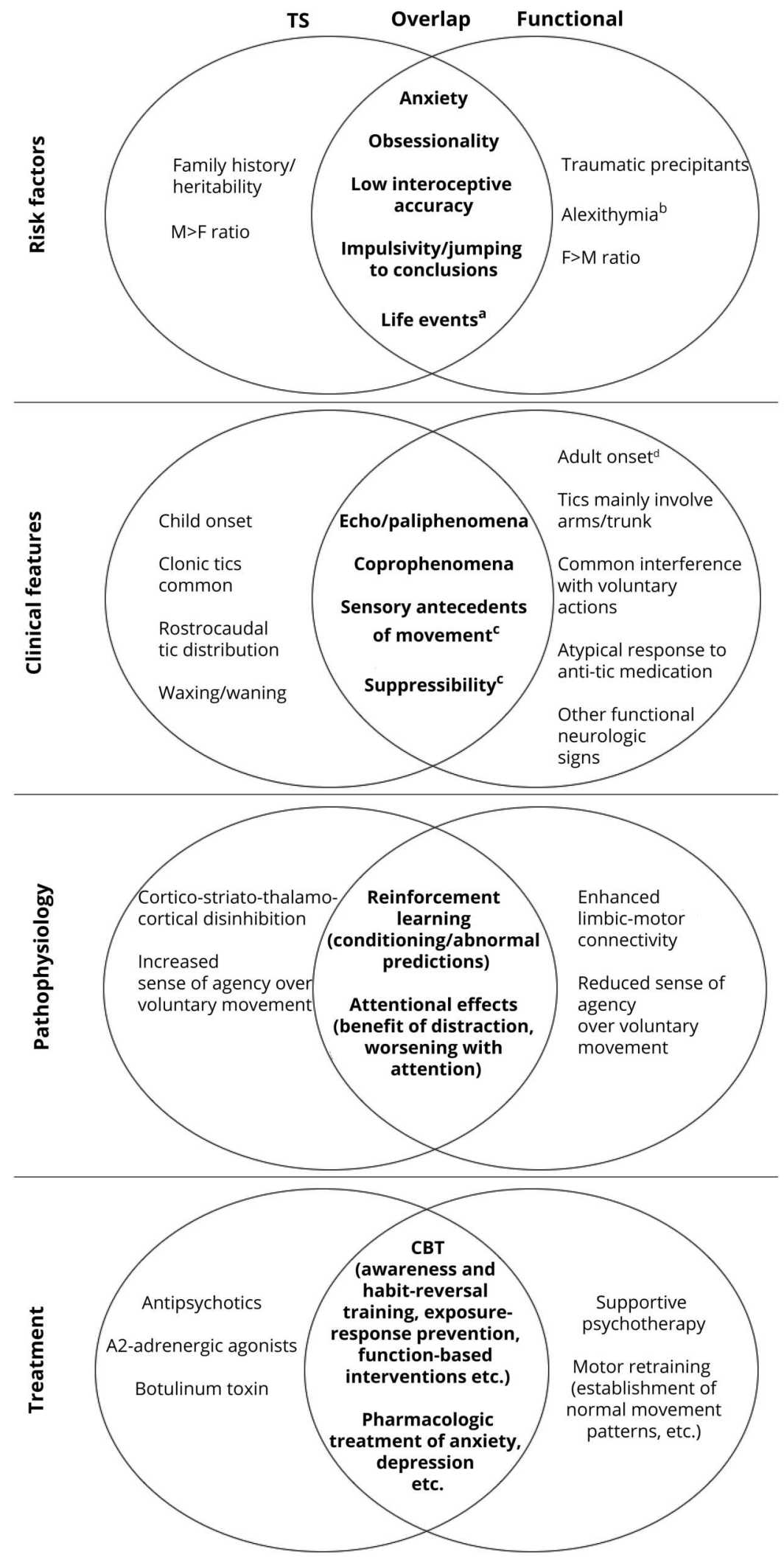

Schematic Venn diagram of risk factors, clinical features, pathophysiologic, and treatment characteristics that are more prevalent as part of tics in TS (nonoverlapping section of the left circle), more prevalent as part of functional tic-like movements (nonoverlapping section of the right circle), and those that often overlap with fea-

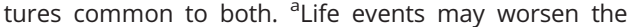
presence of either tics or functional tic-like movements, but are not associated with first-ever tic onset. ${ }^{\mathrm{b}}$ Patients with autism spectrum disorder are often alexithymic and also often present with tics. 'Less commonly reported in functional tic-like movements, but possible. Qualitative descriptions of sensory antecedents of abnormal motor output differ between tics and functional tic-like movements. Voluntary inhibition of functional tic-like movements is often associated with unusual experiences, such as dizziness or malaise. ${ }^{\mathrm{d}}$ Onset of functional tic-like movements may also occur in childhood. TS = Tourette syndrome. $\mathrm{CBT}=$ Cognitive Behavioral Therapy

final approval; and study supervision. D. Martino and A.J. Espay: drafting/revising the manuscript and accept responsibility for conduct of research and will give final approval. A.E. Lang: drafting/revising the manuscript; accepts responsibility for conduct of research and will give final approval; and study supervision. K.P. Bhatia: drafting/revising 
the manuscript; data acquisition; study concept or design; analysis or interpretation of data; and accepts responsibility for conduct of research and will give final approval. M.J. Edwards: drafting/revising the manuscript; study concept or design; accepts responsibility for conduct of research and will give final approval; and study supervision.

\section{Study funding}

Grant support from the VolkswagenStiftung (Freigeist Fellowship).

\section{Disclosure}

C. Ganos holds research grants from the VolkswagenStiftung (Freigeist Fellowship) and the German Parkinson Society and was also supported by the Deutsche Forschungsgemeinschaft (DFG; GA2031/1-1 and GA2031/12) and Actelion Pharmaceuticals. He has also received honoraria from the Movement Disorders Society and Actelion Pharmaceuticals for educational events. D. Martino has received grant support from the Parkinson Association of Alberta, the EU FP-7 Program, and the Immunitaet und Seele Foundation; personal compensation as a consultant/ scientific advisory board member for Sunovion and Allergan, Inc.; publishing royalties from Springer and Oxford University Press; and honoraria from the World Federation of Neurology and the Movement Disorders Society. A.J. Espay has received grant support from the NIH, Great Lakes Neurotechnologies, and the Michael J. Fox Foundation; personal compensation as a consultant/scientific advisory board member for AbbVie, Adamas, Acadia, Acorda, Neuroderm, Impax, Sunovion, Lundbeck, Osmotica Pharmaceutical, and US WorldMeds; publishing royalties from Lippincott Williams \& Wilkins, Cambridge University Press, and Springer; and honoraria from US WorldMeds, Lundbeck, Acadia, Sunovion, the American Academy of Neurology, and the Movement Disorders Society. A.E. Lang has served as an advisor for AbbVie, Acorda, Biogen, Intracellular, Lundbeck, Sun Pharma, Kallyope, Retrophin, Paladin, Seelos, Theravance, Roche, and Corticobasal Degeneration Solutions; has received honoraria from Jazz Pharma, PhotoPharmics, and Sunovion; has received grants from Brain Canada, Canadian Institutes of Health Research, Corticobasal Degeneration Solutions, Edmond J Safra Philanthropic Foundation, Michael J. Fox Foundation, the Ontario Brain Institute, Parkinson Foundation, Parkinson Society Canada, and W. Garfield Weston Foundation; and has received publishing royalties from Elsevier, Saunders, Wiley-Blackwell, Johns Hopkins Press, and Cambridge University Press. K.P. Bhatia holds research grants from EU Horizon 2020 and has received honoraria to speak at meetings or attend advisory boards from Ipsen, Cavion, Allergan, Teva Lundbeck, and Orion pharmaceutical companies. He also receives royalties from Oxford University Press and a stipend for MDCP editorship. M.J. Edwards has received honoraria for educational events from Merz Pharma and Boehringer, outside the submitted work. He holds grants from the Medical Research Council and NIHR. He receives royalties from Oxford University Press. Go to Neurology.org/ $\mathrm{N}$ for full disclosures.

\section{Publication history}

Received by Neurology March 21, 2019. Accepted in final form July 31, 2019.

\section{References}

1. Obeso JA, Rothwell JC, Marsden CD. The neurophysiology of Tourette syndrome. Adv Neurol 1982;35:105-114.

2. Kushner HI. Freud and the diagnosis of Gilles de la Tourette's illness. Hist Psychiatry 1998;9:1-25.

3. Paszek J, Pollok B, Biermann-Ruben K, et al. Is it a tic? Twenty seconds to make a diagnosis. Mov Disord 2010;25:1106-1108.

4. Baizabal-Carvallo JF, Jankovic J. The clinical features of psychogenic movement disorders resembling tics. J Neurol Neurosurg Psychiatry 2014;85:573-575.

5. Demartini B, Ricciardi L, Parees I, Ganos C, Bhatia KP, Edwards MJ. A positive diagnosis of functional (psychogenic) tics. Eur J Neurol 2015;22:527-e36.

6. Kutlubaev MA, Xu Y, Hackett ML, Stone J. Dual diagnosis of epilepsy and psychogenic nonepileptic seizures: systematic review and meta-analysis of frequency, correlates, and outcomes. Epilepsy Behav 2018;89:70-78.

7. Stone J, Carson A, Duncan R, et al. Which neurological diseases are most likely to be associated with symptoms unexplained by organic disease. J Neurol 2012;259:33-38.

8. Ganos C, Erro R, Cavanna AE, Bhatia KP. Functional tics and echophenomena. Parkinsonism Relat Disord 2014;20:1440-1441.

9. Ganos C, Edwards MJ, Muller-Vahl K. "I swear it is Tourette's!": on functional coprolalia and other tic-like vocalizations. Psychiatry Res 2016;246:821-826.

10. Roberts NA, Reuber M. Alterations of consciousness in psychogenic nonepileptic seizures: emotion, emotion regulation and dissociation. Epilepsy Behav 2014;30: 43-49.

11. Van Hulle CA, Schmidt NL, Goldsmith HH. Is sensory over-responsivity distinguishable from childhood behavior problems? A phenotypic and genetic analysis. J Child Psychol Psychiatry 2012;53:64-72.

12. Maia TV, Conceicao VA. The roles of phasic and tonic dopamine in tic learning and expression. Biol Psychiatry 2017;82:401-412.

13. Leckman JF, Riddle MA. Tourette's syndrome: when habit-forming systems form habits of their own? Neuron 2000;28:349-354.

14. Yael D, Vinner E, Bar-Gad I. Pathophysiology of tic disorders. Mov Disord 2015;30: 1171-1178.

15. Bronfeld M, Yael D, Belelovsky K, Bar-Gad I. Motor tics evoked by striatal disinhibition in the rat. Front Syst Neurosci 2013;7:50.

16. McCairn KW, Bronfeld M, Belelovsky K, Bar-Gad I. The neurophysiological correlates of motor tics following focal striatal disinhibition. Brain 2009;132: $2125-2138$

17. McCairn KW, Nagai $Y$, Hori $Y$, et al. A primary role for nucleus accumbens and related limbic network in vocal tics. Neuron 2016;89:300-307.

18. Israelashvili M, Bar-Gad I. Corticostriatal divergent function in determining the temporal and spatial properties of motor tics. J Neurosci 2015;35: $16340-16351$.

19. Kataoka Y, Kalanithi PS, Grantz H, et al. Decreased number of parvalbumin and cholinergic interneurons in the striatum of individuals with Tourette syndrome. J Comp Neurol 2010;518:277-291.

20. Voon V, Cavanna AE, Coburn K, Sampson S, Reeve A, LaFrance WC Jr. Functional neuroanatomy and neurophysiology of functional neurological disorders (conversion disorder). J Neuropsychiatry Clin Neurosci 2016;28:168-190.

21. Edwards MJ, Adams RA, Brown H, Parees I, Friston KJ. A Bayesian account of "hysteria". Brain 2012;135:3495-3512.

22. Bodde NM, Brooks JL, Baker GA, et al. Psychogenic non-epileptic seizuresdefinition, etiology, treatment and prognostic issues: a critical review. Seizure 2009; 18:543-553.

23. Misirlisoy E, Brandt V, Ganos C, Tubing J, Munchau A, Haggard P. The relation between attention and tic generation in Tourette syndrome. Neuropsychology 2015; 29:658-665.

24. Edwards MJ, Fotopoulou A, Parees I. Neurobiology of functional (psychogenic) movement disorders. Curr Opin Neurol 2013;26:442-447.

25. Pappert EJ, Goetz CG, Louis ED, Blasucci L, Leurgans S. Objective assessments of longitudinal outcome in Gilles de la Tourette's syndrome. Neurology 2003;61: 936-940.

26. Lang AE, Consky E, Sandor P. "Signing tics" - insights into the pathophysiology of symptoms in Tourette's syndrome. Ann Neurol 1993;33:212-215.

27. Kahl U, Schunke O, Schöttle D, et al. Tic phenomenology and tic awareness in adults with autism. Movement Disord Clin Pract 2015;2:237-242.

28. van der Salm SM, Tijssen MA, Koelman JH, van Rootselaar AF. The bereitschaftspotential in jerky movement disorders. J Neurol Neurosurg Psychiatry 2012;83: $1162-1167$.

29. Houdayer E, Walthall J, Belluscio BA, Vorbach S, Singer HS, Hallett M. Absent movement-related cortical potentials in children with primary motor stereotypies. Mov Disord 2014;29:1134-1140.

30. Stenner MP, Haggard P. Voluntary or involuntary? A neurophysiologic approach to functional movement disorders. Handb Clin Neurol 2016;139:121-129. 
31. Edwards MJ, Moretto G, Schwingenschuh P, Katschnig P, Bhatia KP, Haggard P. Abnormal sense of intention preceding voluntary movement in patients with psychogenic tremor. Neuropsychologia 2011;49:2791-2793.

32. Moretto G, Schwingenschuh P, Katschnig P, Bhatia KP, Haggard P. Delayed experience of volition in Gilles de la Tourette syndrome. J Neurol Neurosurg Psychiatry 2011;82:1324-1327.

33. Ganos C, Asmuss L, Bongert J, Brandt V, Munchau A, Haggard P. Volitional action as perceptual detection: predictors of conscious intention in adolescents with tic disorders. Cortex 2015;64:47-54.

34. Kranick SM, Moore JW, Yusuf N, et al. Action-effect binding is decreased in motor conversion disorder: implications for sense of agency. Mov Disord 2013;28: $1110-1116$.

35. Delorme C, Salvador A, Voon V, et al. Illusion of agency in patients with gilles de la Tourette syndrome. Cortex 2016;77:132-140.

36. Rae CL, Critchley HD, Seth AK. A bayesian account of the sensory-motor interactions underlying symptoms of Tourette syndrome. Front Psychiatry 2019;10:29.

37. Delorme C, Salvador A, Valabregue R, et al. Enhanced habit formation in Gilles de la Tourette syndrome. Brain 2016;139:605-615.

38. Demartini B, Petrochilos P, Ricciardi L, Price G, Edwards MJ, Joyce E. The role of alexithymia in the development of functional motor symptoms (conversion disorder). J Neurol Neurosurg Psychiatry 2014;85:1132-1137.

39. Hirschtritt ME, Lee PC, Pauls DL, et al. Lifetime prevalence, age of risk, and genetic relationships of comorbid psychiatric disorders in Tourette syndrome. JAMA psychiatry 2015;72:325-333.

40. Seppi K, Ray Chaudhuri K, Coelho M, et al. Update on treatments for nonmotor symptoms of Parkinson's disease-an evidence-based medicine review. Mov Disord 2019;34:180-198.

41. Enders L, Spector JT, Altenmuller E, Schmidt A, Klein C, Jabusch HC. Musician's dystonia and comorbid anxiety: two sides of one coin? Mov Disord 2011;26:539-542.

42. Pile V, Robinson S, Topor M, Hedderly T, Lau JYF. Attention bias for social threat in youth with tic disorders: links with tic severity and social anxiety. Child Neuropsychol 2019;25:394-409.

43. Steinberg T, Shmuel-Baruch S, Horesh N, Apter A. Life events and Tourette syndrome. Compr Psychiatry 2013;54:467-473.

44. Stone J, Carson A, Aditya $\mathrm{H}$, et al. The role of physical injury in motor and sensory conversion symptoms: a systematic and narrative review. J Psychosom Res 2009;66: 383-390.
45. Eddy CM, Cavanna AE. Triangles, tricks and tics: hyper-mentalizing in response to animated shapes in Tourette syndrome. Cortex 2015;71:68-75.

46. Kinnaird E, Stewart C, Tchanturia K. Investigating alexithymia in autism: a systematic review and meta-analysis. Eur Psychiatry 2019;55:80-89.

47. Ganos C, Garrido A, Navalpotro-Gomez I, et al. Premonitory urge to tic in Tourette's is associated with interoceptive awareness. Mov Disord 2015;30: 1198-1202.

48. Ricciardi L, Demartini B, Crucianelli L, Krahe C, Edwards MJ, Fotopoulou A. Interoceptive awareness in patients with functional neurological symptoms. Biol Psychol 2016;113:68-74.

49. Eddy CM, Cavanna AE. Do patients with Tourette syndrome jump to conclusions? J Neuropsychiatry Clin Neurosci 2014;26:396-399.

50. Parees I, Kassavetis P, Saifee TA, et al. "Jumping to conclusions" bias in functional movement disorders. J Neurol Neurosurg Psychiatry 2012;83:460-463.

51. Worbe Y, Mallet L, Golmard JL, et al. Repetitive behaviours in patients with Gilles de la Tourette syndrome: tics, compulsions, or both? PLoS One 2010;5:e12959.

52. Stamelou M, Cossu G, Edwards MJ, et al. Familial psychogenic movement disorders. Mov Disord 2013;28:1295-1298.

53. Martino D, Pringsheim TM. Tourette syndrome and other chronic tic disorders: an update on clinical management. Expert Rev Neurother 2018;18:125-137.

54. Nielsen G, Stone J, Matthews A, et al. Physiotherapy for functional motor disorders: a consensus recommendation. J Neurol Neurosurg Psychiatry 2015;86:1113-1119.

55. Piacentini J, Woods DW, Scahill L, et al. Behavior therapy for children with Tourette disorder: a randomized controlled trial. JAMA 2010;303:1929-1937.

56. Sukhodolsky DG, Woods DW, Piacentini J, et al. Moderators and predictors of response to behavior therapy for tics in Tourette syndrome. Neurology 2017;88: $1029-1036$.

57. Kurlan R, Deeley C, Como PG. Psychogenic movement disorder (pseudo-tics) in a patient with Tourette's syndrome. J Neuropsychiatry Clin Neurosci 1992;4: 347-348.

58. Robinson S, Hedderly T. Novel psychological formulation and treatment of "tic attacks" in Tourette syndrome. Front Pediatr 2016;4:46.

59. Zekaj E, Saleh C, Porta M, Servello D. Temporary deep brain stimulation in Gilles de la Tourette syndrome: a feasible approach? Surg Neurol Int 2015;6:122.

60. Espay AJ, Schwarzschild MA, Tanner CM, et al. Biomarker-driven phenotyping in Parkinson's disease: a translational missing link in disease-modifying clinical trials. Mov Disord 2017;32:319-324. 


\section{Neurology}

\section{Tics and functional tic-like movements: Can we tell them apart? \\ Christos Ganos, Davide Martino, Alberto J. Espay, et al. \\ Neurology published online September 24, 2019 \\ DOI 10.1212/WNL.0000000000008372}

This information is current as of September 24, 2019

\section{Updated Information \&} Services

Subspecialty Collections

\section{Permissions \& Licensing}

Reprints including high resolution figures, can be found at: http://n.neurology.org/content/early/2019/09/24/WNL.0000000000008 372.full

This article, along with others on similar topics, appears in the following collection(s):

\section{Conversion}

http://n.neurology.org/cgi/collection/conversion

Motor Control

http://n.neurology.org/cgi/collection/motor_control

Tics

http://n.neurology.org/cgi/collection/tics

Tourette syndrome

http://n.neurology.org/cgi/collection/tourette_syndrome

Information about reproducing this article in parts (figures,tables) or in its entirety can be found online at:

http://www.neurology.org/about/about_the_journal\#permissions

Information about ordering reprints can be found online:

http://n.neurology.org/subscribers/advertise

Neurology ${ }^{\circledR}$ is the official journal of the American Academy of Neurology. Published continuously since 1951, it is now a weekly with 48 issues per year. Copyright (O 2019 American Academy of Neurology. All rights reserved. Print ISSN: 0028-3878. Online ISSN: 1526-632X.

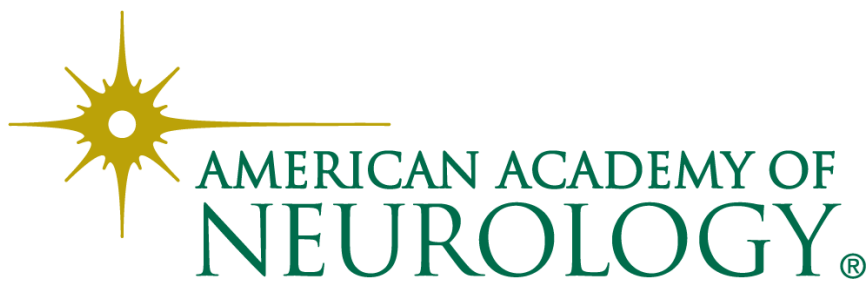

